\title{
Valoración de Ecuaciones derivadas del Flujo Pulmonar y Regurgitación Tricuspídea: Utilidad en el Test de Vasoreactividad Pulmonar
}

\author{
Tania Muñoz ${ }^{1 *}$, Simón Tovar ${ }^{1}$, Elizabeth Hirschhaut ${ }^{1}$, Mariangel Millán ${ }^{1}$, Yvette Betancourt ${ }^{1 a}$, Katiusca Arteaga. ${ }^{1 a}$ \\ 1Departamento de Cardiología, Hospital Militar Dr. Carlos Arvelo, Caracas, Venezuela. \\ a Técnico ecocardiografista
}

Financiamiento: para realizar este trabajo científico se utilizaron los recursos del Hospital.

Antecedentes: La presión media arterial pulmonar (PMAP) es una variable hemodinámica indispensable para el diagnóstico, clasificación y pronóstico de la Hipertensión Pulmonar (HP). Su cuantificación se realiza en forma invasiva por cateterismo cardíaco derecho (CCD) y no invasivamente por ecocardiografía Doppler. Masuyama propuso su medición mediante el gradiente transvalvular pulmonar diastólico derivado de la velocidad máxima inicial de la regurgitación pulmonar $(\Delta \mathrm{RPi} 2)$ correspondiendo cercanamente a la medición invasiva.

Objetivos: Revalidar 3 métodos ecocardiográficos que estiman la PMAP y valorar la utilidad del método de Chemla en el Test de Reactividad Vascular Pulmonar (TRVP).

Métodos: Estudio prospectivo, observacional, doble ciego divido en dos etapas. A) o I) 30 pacientes se realizó ecocardiografía Doppler diagnóstica en nuestro centro. Se midieron regurgitación tricuspídea (RT) y tiempo de aceleración pulmonar (TAP) para derivar las siguientes ecuaciones: 1) 0.61xPSAP+1.95 (Chemla)
2) Gradiente presión media RT $(\triangle \mathrm{PmRT})+\mathrm{PAD}$ (presión-aurícula derecha) (Aduen) y 3) 79-0.45xTAP o 90-0.60xTAP, según sea el valor del TAP. B) o II) 10 pacientes enrolados para realizar el TRVP comparando la medición ecocardiográfica (Chemla) con CCD.

Resultados: En la primera parte del estudio se encontró alta correlación entre las 3 ecuaciones: ChemlaAduen, $\quad \mathrm{R}^{2}=0.91 ; \quad$ Chemla-Kitabatake, $\quad \mathrm{R}^{2}=0.87$; Aduen-Kitabatake, $R^{2}=0,91$. En la segunda parte comparando la PMAP-Chemla y Cateterismo derecho (CD) obtuvimos alta correlación: en tiempo 0,30 min y recuperación: $\left(R^{2}=0.87,0.99,0.98\right.$, respectivamente $)$. Ambas partes del estudio mostraron límites de concordancia satisfactoria con valor medio de la diferencia entre los métodos cercano a 1 en el t30 y tR del TRVP.

Conclusión: los métodos dependientes de la medición de la RT son efectivos y confiables para estimar la PMAP. El método de Chemla es útil en el TRVP.

Palabras claves: Ecocardiografía doppler; tiempo de aceleración pulmonar; Cateterismo cardíaco derecho; Hipertensión pulmonar. 


\section{Valuation of equations derived from Pulmonary Flow and Tricuspid Regurgitation: usefulness in Pulmonary Vasoreactivity Testing.}

Background: Mean Pulmonary arterial pressure (PMAP)is an indispensable hemodynamic variable for the diagnosis, classification and prognosis of Pulmonary Hypertension $(\mathrm{PH})$. Its quantification is performed invasively by cardiac catheterization and non-invasively by Doppler echocardiography. Masuyama proposed its measurement by the transvalvular diastolic pulmonary gradient derived from the initial maximum velocity of pulmonary regurgitation( $\triangle \mathrm{PRi} 2)$ corresponding closely to the invasive measurement.

Objectives: to compare 3 known echocardiographic methods to estimate MPAP and demonstrate the usefulness of the Chemla method in the Pulmonary Vascular Reactivity Test (PVRT).

Methods: prospective, observational, double-blind study divided into two stages. A) 30 patients underwent diagnostic Doppler echocardiography. Tricuspid regurgitation (TR) and pulmonary acceleration time (PAT) were measured to derive the equations: 1 ) $0.61 \mathrm{xSPAP}+1.95$ (Chemla) 2) Gradient mean pressure TR $(\triangle \mathrm{PmTR})+\mathrm{RAP}$ (right atrium pressure)
(Aduen).3) 79-0.45xPAT o 90-0.60xPAT depending on the value of PAT. B) 10 patients enrolled to PVRT comparing the echocardiographic measurement (Chemla) with right catheterization.

Results: in the first part of the study a high correlation between the 3 equations was found : ChemlaAduen, $\mathrm{R}^{2}=0.91$; Chemla-Kitabatake, $\mathrm{R}^{2}=0.87$; Aduen-Kitabatake, $\mathrm{R}^{2}=0.91$. In the second part comparing the MPAP-Chemla and RHC we obtained a high correlation in time $0,30 \mathrm{~min}$ and recovery: $\left(\mathrm{R}^{2}=0.87,0,99,0.98\right.$, respectively). Both parts of the study showed satisfactory limits concordance with mean value of the difference between the methods close to 1 in the $t 30$ and $t R$ of the TRVP.

Conclusion: the methods dependent on the measurement of the TR are effective and reliable for estimating MPAP. The Chemla method is useful in the PVRT

Keywords: doppler echocardiography; pulmonary acceleration time; right heart catheterization; pulmonary hypertension. 


\section{Introducción:}

La HP es diagnosticada por la medición de la PMAP idóneamente desde la etapa más temprana. La elevación sostenida de la post-carga es impuesta por los diferentes mecanismos de hipertrofia de la pared muscular, engrosamiento y fibrosis endotelial, proliferación de la adventicia con necrosis fibrinoide, estado pro-inflamatorio y trombogénico, condicionando la isquemia y apoptosis de los vasos sanguíneos pulmonares independiente de la etiología de la enfermedad ${ }^{1-3}$ La elevación de la PMAP es estimada con más confianza invasivamente; sin embargo, diferentes autores han canalizado esfuerzos para ofrecer su medición no invasiva con éxito a partir de diferentes ecuaciones derivadas de ecocardiografía doppler. Por mucho tiempo ha sido método de referencia la determinación del $\Delta \mathrm{RPi}^{2}$ evaluable en $80 \%$ de los casos ${ }^{4}$. Hace años Kitabatake et $\mathrm{al}^{5}$, sugirieron estimar PMAP por medio del cálculo del TAP, complementado con el estudio de la morfología del flujo pulmonar. Chemla et $\mathrm{al}^{6}$, propusieron un método derivado a partir de la velocidad máxima de la RT y cuantificación de la Presión sistólica arterial pulmonar (PSAP) con la ecuación de Bernoulli modificada obteniendo valores cercanos a los obtenidos invasivamente. Otra ecuación derivada de la RT, es a partir de la estimación del gradiente medio de presión $(\triangle \mathrm{PmRT})$ y la PAD, enunciada por Aduen et al ${ }^{7}$. No hay estudios que reporten la utilidad de estos métodos en el TRVP.

\section{Métodos:}

Estudio prospectivo, doble ciego, observacional que se dividió en dos etapas. En la primera parte se enrolaron 30 pacientes con indicación de ecocardiografía Doppler diagnóstico realizado en nuestra Unidad de estudios ecocardiográficos durante el período comprendido entre 14 de enero y 14 de febrero del 2019. No se hizo distinción por grupo etario, sexo, comorbilidades, ni se omitió la medicación. No hubo criterios de exclusión. Las características clínicas, hemodinámicas y demográficas se muestran en la Tabla 1. En la segunda parte del estudio se incluyeron 11 pacientes referidos de la consulta de Neumología, Cardiología Infantil y Cardiolo-

\begin{tabular}{|c|c|c|}
\hline SEXO (M/F) & & $17 / 13$ \\
\hline EDAD media (RANGO) años & 58 & $(17-84)$ \\
\hline Fracción de eyección media (RANGO)(\%) & 57 & (8-78) \\
\hline Presión sistólica arterial pulmonar media (RANGO) mmHg & 31 & (18-72) \\
\hline DVD media (RANGO) mm & 28 & $(19-46)$ \\
\hline TAP & 125 & $(80-147)$ \\
\hline VRT media (RANGO) m/s & 2,42 & $(1,74-3,78)$ \\
\hline Gmed RT media (RANGO) mmHg & 16,35 & $(8,21-32)$ \\
\hline RVP media (RANGO) UW & 2,73 & $(1,12-8,35)$ \\
\hline PAD media (RANGO) mm & 6,8 & $(5-20)$ \\
\hline \multicolumn{3}{|l|}{ DIAGNOSTICOS: } \\
\hline HAS & & 11 \\
\hline Cardiopatía mixta HAS+ isq & & 5 \\
\hline Cardiopatia hipertensiva en FD & & 2 \\
\hline CIC en FD & & 1 \\
\hline Dolor torácico de EAP & & 2 \\
\hline Palpitaciones en estudio & & 2 \\
\hline Endocarditis bacteriana & & 1 \\
\hline Dilatación Aorta ascendente & & 1 \\
\hline EPOC & & 2 \\
\hline Derrame pericárdico & & 1 \\
\hline CIC: EACO de 1 vaso & & 1 \\
\hline Pte sano & & 1 \\
\hline
\end{tabular}




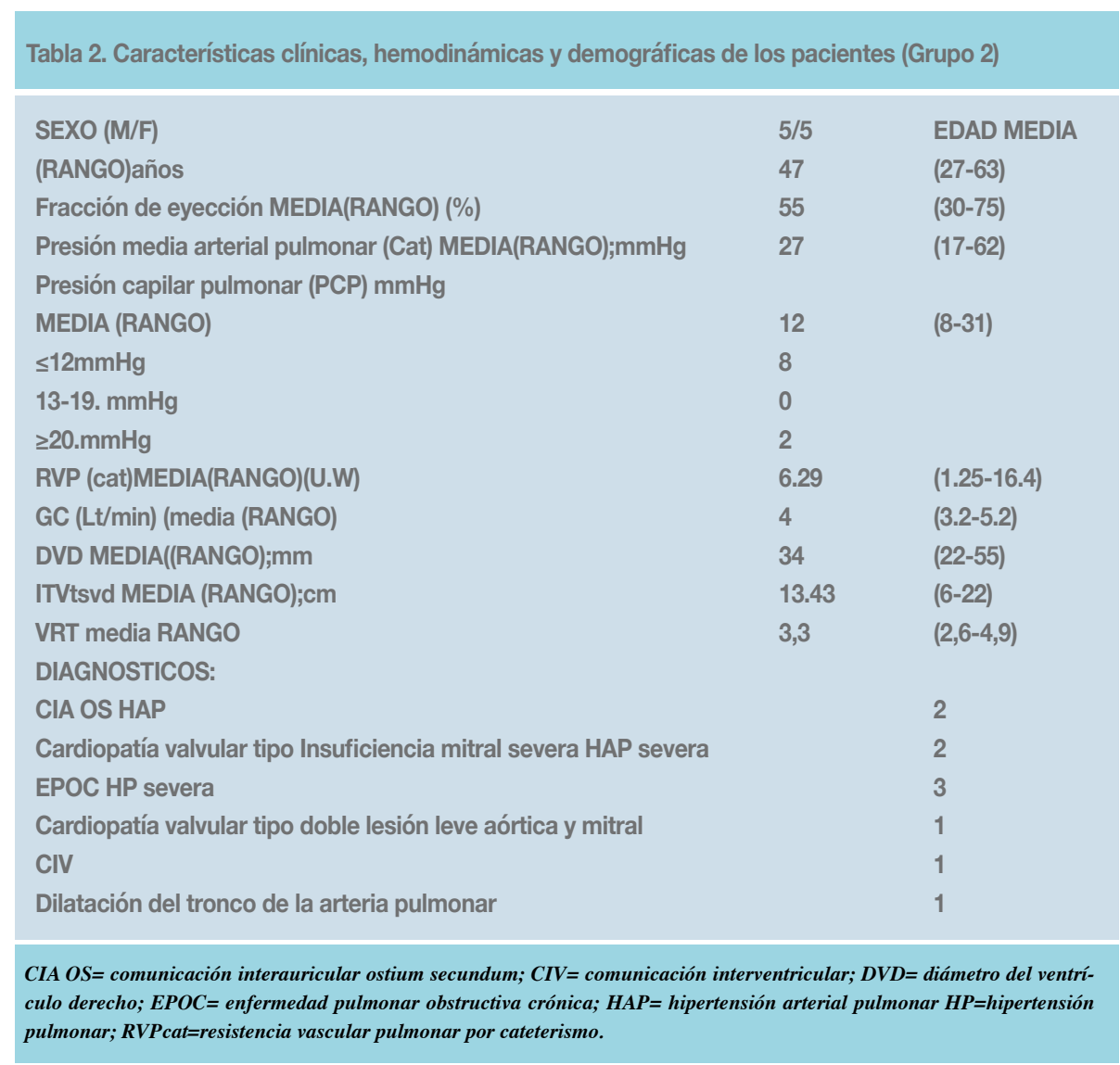

gía del mismo centro con diagnóstico presuntivo de HP, de diferentes etiología, grado de severidad y criterios ecocardiográficos, para realizar el test de reactividad vascular pulmonar. Estos pacientes fueron estudiados de julio a diciembre del 2009. Criterios de exclusión: pacientes con inestabilidad hemodinámica, hipoxemia crónica o aguda, trastornos de coagulación. Un paciente fue retirado del estudio por la imposibilidad de realizar las mediciones con el catéter y en 2 pacientes solo se hicieron las mediciones iniciales por la importante mejoría hemodinámica pulmonar que presentaron con el tratamiento, respecto al ecocardiograma de referencia. Las características clínicas, hemodinámicas y demográficas se muestran en la Tabla 2.

Todos los pacientes firmaron consentimiento informado. El estudio fue aprobado por el Comité de Bioética y Ética Médica del Hospital.

Ecocardiografía: Las mediciones Doppler y bidimensional se realizaron con los equipos Philips Sonos 7500 S3 y Philips iE33 SE1 de acuerdo a las guías de la So- ciedad Americana de Ecocardiografía ${ }^{8.9} \cdot$ El TAP (m/s) se obtuvo con señal de onda de pulso del flujo pulmonar anterógrado proximal a la válvula pulmonar en la vista del eje corto paraesternal a nivel de grandes vasos. El volumen de muestra Doppler se colocó justo antes de cierre de la válvula Se midió el tiempo de aceleración del tracto de salida del ventrículo derecho (TSVD) desde el comienzo hasta la velocidad máxima del flujo ${ }^{10}$. La ecuación derivada para obtener el cálculo de la PMAP (Kitabatake) dependerá de su valor. Si TAP $>120,1$.PMAP=79-0.45x$\mathrm{TAP}^{11}$. PMAP=90-0.60xTAP ${ }^{12}$. La VRT (m/s) se obtuvo con Doppler continuo colocado en el flujo regurgitante tricuspídeo a nivel valvular. Se evaluó también en la vista de 4 cámaras con la intención de obtener la máxima velocidad posible. Con esta y la PAD, empleando la ecuación de modificada de Bernoulli se obtuvieron los valores de PSAP $^{11,13}$ para luego calcular la PMAP con la ecuación propuesta por $\mathrm{Chemla}^{7}=0.61 \times \mathrm{XSAP}+1.95 \mathrm{El} \Delta \mathrm{PmRT}$ se evaluó con Doppler continuo rastreando el flujo regurgitante tricuspídeo. La diferencia de presión media se mide a partir de la integral tiempo-velocidad (ITV) ${ }^{10}$. La fór- 
mula propuesta por Aduen es $\mathrm{PMAP}=\Delta \mathrm{PmRT}+\mathrm{PAD} . \mathrm{La}$ FE (fracción de eyección) se determinó por el método de Simpson en la vista de 4 cámaras.

En ambas partes del trabajo, las mediciones fueron realizadas 3 veces y se promediaron.

Cateterismo cardíaco derecho: En la Unidad de Hemodinamia, a los pacientes del segundo grupo (10) se les colocó Catéter de Swan Ganz arrow, 6 o 7 French (F) para obtener las mediciones de presiones y flujos pulmonares. Se empleó la técnica de Seldinger para el abordaje de la vena yugular interna (VYI) o subclavia ${ }^{14}$. El gasto cardíaco (GC) se determinó por la técnica de termodilución y la RVP con la ecuación: RVP=PMAP-PCP/GC. La mediciones de PMAP; PSAP y presión diastólica arteria pulmonar (PDAP) las realizó automáticamente el equipo. La PCP se obtuvo por acuñamiento del balón. ${ }^{14-16}$

Análisis estadístico: Se utilizó software MedCalc statitic 2019 versión 18.11.3/ 14.0 de SPSS. Se realizó análisis de regresión lineal de la PMAP entre el método invasivo y no invasivo (Método de Chemla et al). También entre los métodos de Chemla, Aduen y Kitabatake. Se determinó el Coeficiente de correlación de Pearson en todos los casos y se derivó una ecuación de regresión. Los valores calculados luego se estudiaron utilizando el análisis de Bland-Altman para determinar los límites de concordancia, DE y promedio de las diferencias entre los métodos en ambas partes del estudio. Las imágenes fueron reevaluadas para cuantificar la confiabilidad del intraobservador e interobservador.

\section{Resultados:}

Las características de los pacientes del primer grupo ${ }^{30}$ se muestran en la Tabla 1. El promedio de PSAP fue 31(1872) y TAP de 125(80-147). Once pacientes presentaron diagnóstico de Hipertensión Arterial sistémica (HAS) y 5 Cardiopatía Mixta: isquémica e hipertensiva. El análisis de correlación lineal entre los 3 métodos reportó lo siguiente: Chemla-Aduen, $\mathrm{R}^{2}=0,91$; Chemla-Kitabatake $\mathrm{R}^{2}=0,87$; Kitabatake-Aduen $\mathrm{R}^{2}=0,91$ (Figura 1). El Análisis de Bland Altman mostró límites de concordancia satisfactorios entre todos los métodos analizados y el valor medio de las diferencias de la PMAP entre los métodos ecocardiográficos cercano a 1: Chemla-Kitabatake(media-1.27,L:5.72/-8.26, Chemla-Aduen(media-0.22,L:5.71/-6.16); Kitabatake-Aduen (media 1.045;L:9.57/-7.49) (Figura 2). En el segundo grupo analizado (Tabla 2), 9 de ellos presentaron PCP igual o menor de $12 \mathrm{mmHg}$, siendo variado los subgrupos dentro de la cla-
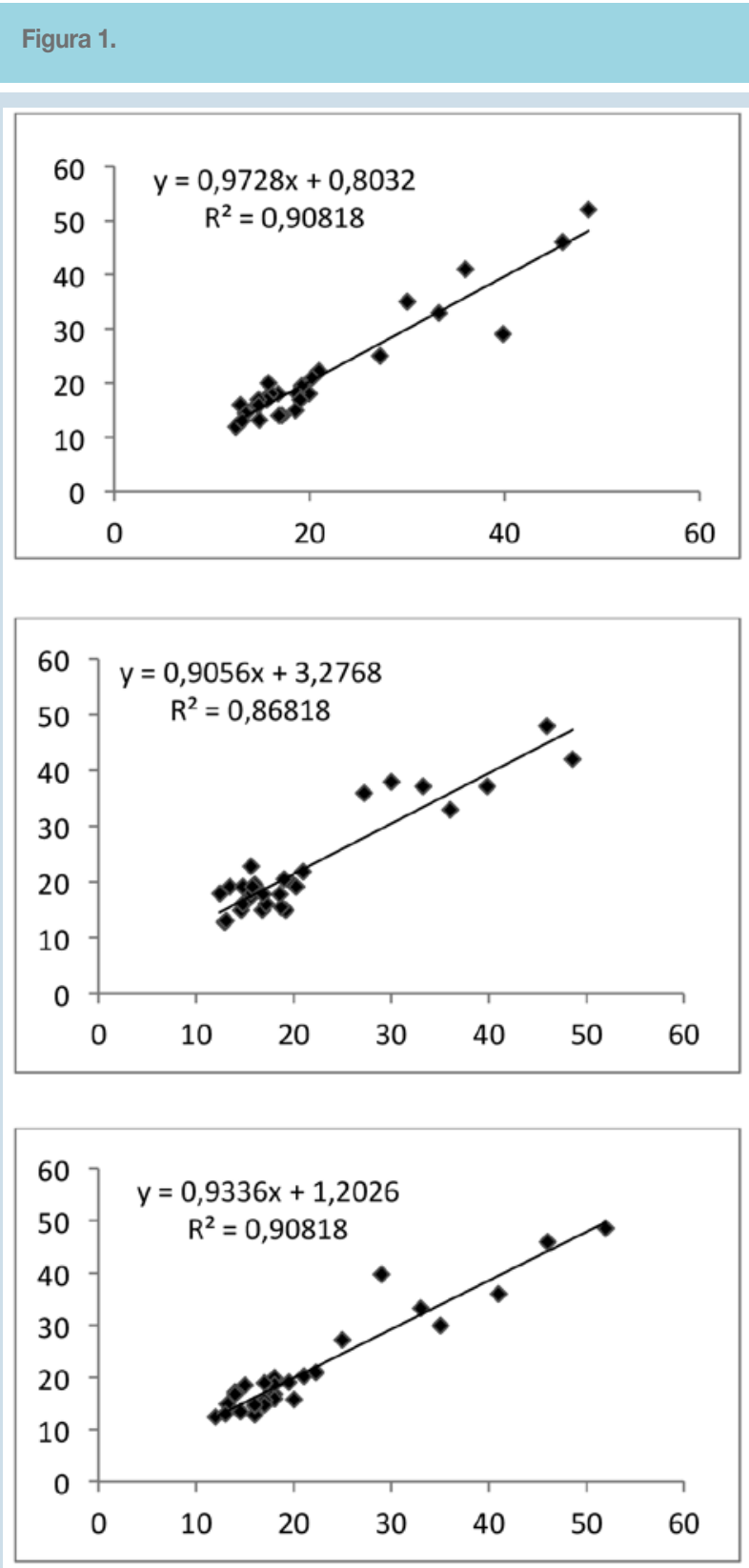

Análisis de regresión lineal comparando los métodos ecocardiográficos Doppler de Chemla-Aduen(arriba),Chemla-Kitabatake(medio) y Kitabatake-Aduen(abajo).

sificación de HP aceptada por SEC: 1.4.4. Cardiopatía congénita: CIA (2); CIV (1). 2.3.Valvulopatías: valvulopatía mitral tipo IM severa (2),Valvulopatía aórtica y mitral tipo doble lesión leve (1); 3.1.EPOC severa + HP severa (3) y 3.5 Enfermedades del desarrollo pulmonar: Dilatación del tronco de la arteria pulmonar (1). En la segunda etapa del estudio el análisis de correlación lineal entre CD y la ecuación de Chemla reportó alta correlación en el t0, t30 y tR $\left(R^{2}=0.87,0,99\right.$ y 0,98 respec- 


\section{Figura 2.}
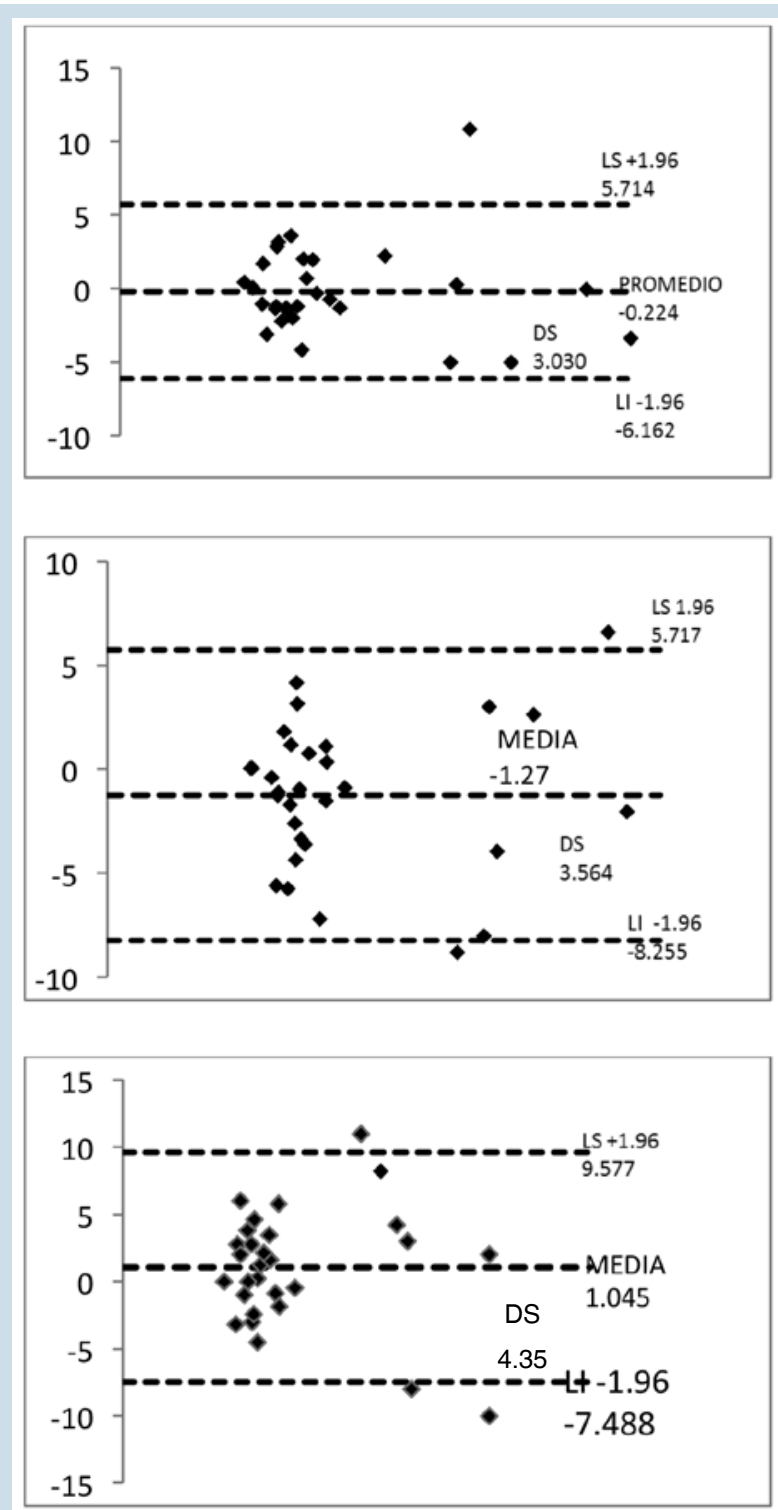

Análisis de Bland Altman comparando los métodos ecocardiográficos Doppler de Chemla-Aduen(arriba),Chemla-Kitabatake(medio) y Kitabatake y Aduen(abajo).

tivamente, I.C. 95 \%) (Figura 3). El análisis de Bland Altman mostró que el valor medio de la diferencia entre el PMAP medido de forma invasiva y no invasiva en el TRVP fue cercano a 1 en el t30 y tR. 1. t0: media-2.1, L:15.25/-11.05; t30: media-0.75,L:2.70/-2.61: tR: media -1,L:4.02/-6.02.Límites de concordancia (valor medio $\pm 1.96 \times \mathrm{SD}$ ) (Figura 4).

\section{Discusión:}

El conocimiento de la presión arterial pulmonar (PAP)
Figura 3.
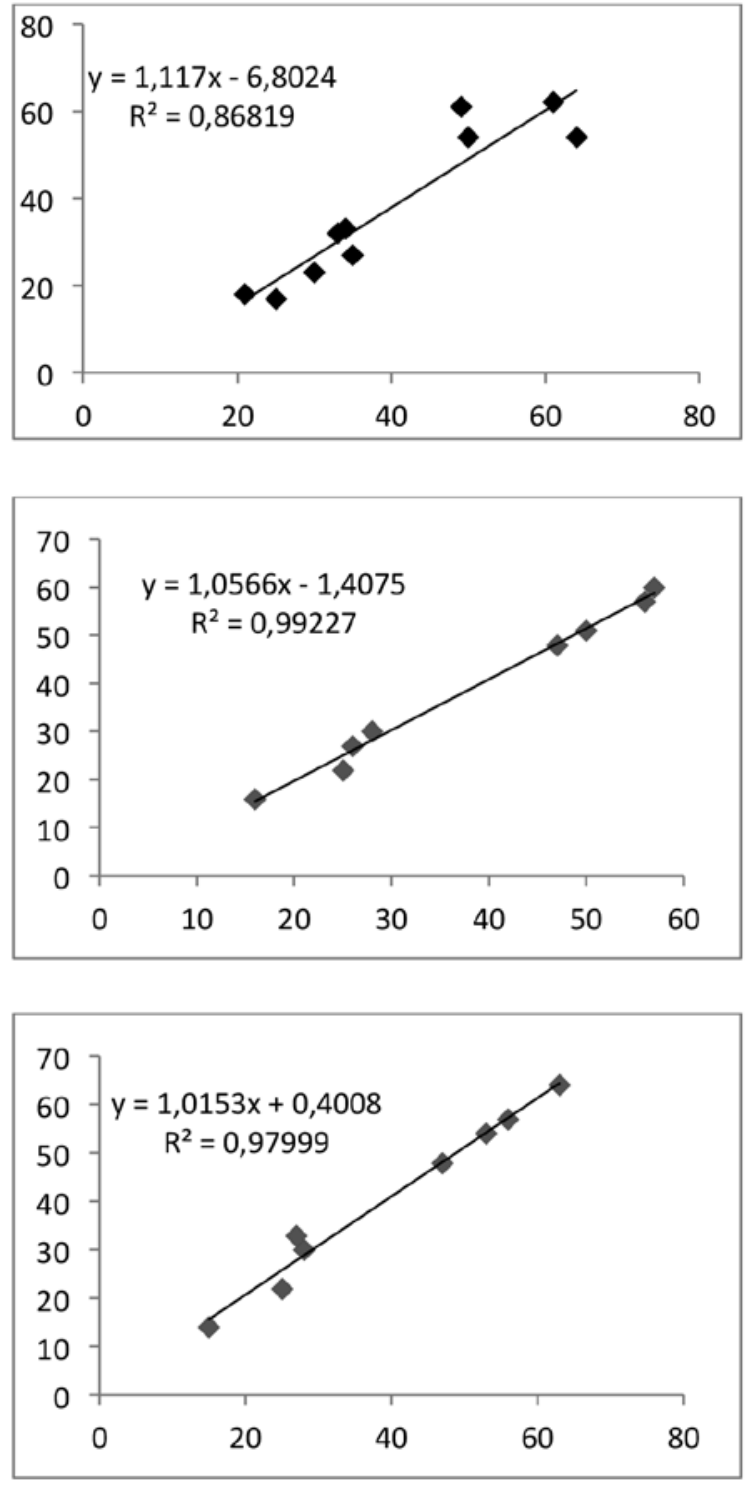

Análisis de regresión lineal comparando la medición invasiva (CatSW) y ecocardiográfica (Chemla et al) de la PMAP en el tiempo 0,30 y Recuperación del TRVP.

es fundamental para el tratamiento de la enfermedad cardíaca. Las mediciones no invasivas se pueden derivar de la interrogación Doppler del tracto de salida del ventrículo derecho (TSVD), la regurgitación tricúspide (TR) y las señales de regurgitación pulmonar $(\mathrm{RP})^{17-25}$. Desde hace más de 20 años tenemos a disposición ecuaciones ecocardiográficas para obtener el cálculo de la PMAP. Kitabatake et $\mathrm{al}^{5}$ demostraron posible su estimación a partir del TAP obtenido con Doppler pulsado en el TVSD 
Figura 4.
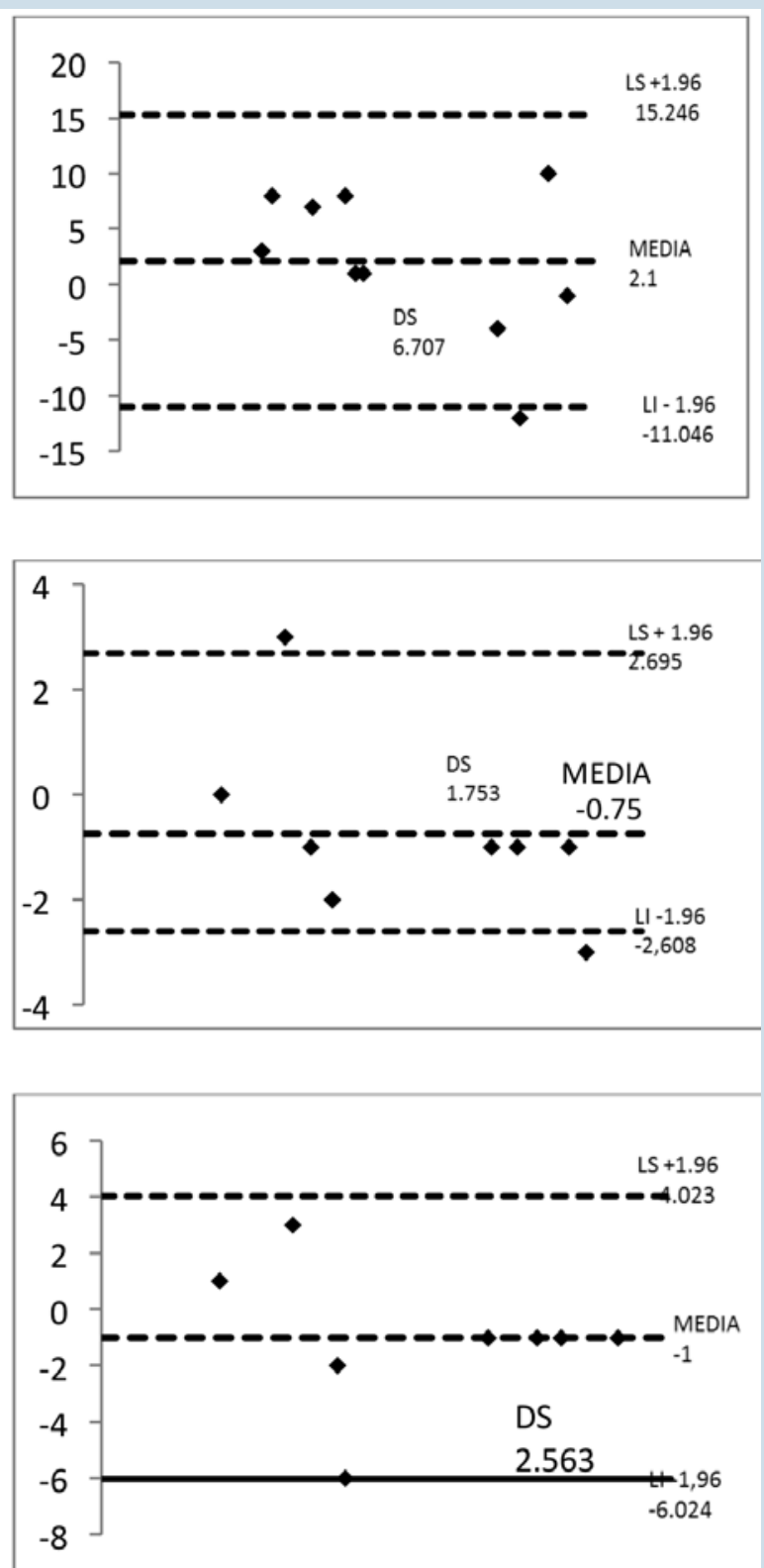

Análisis de Bland Altman mostrando límites de concordancia satisfactorios entre los métodos invasivo y no invasivo (Chemla) en el momento 0,30 y recuperación del TRVP

y describieron diferentes patrones de velocidad de flujo con presencia de muesca mesosistólica en los casos severos de HP. Observaciones posteriores demostraron que frecuencia cardíaca (FC) por fuera del rango normal reduce la efectividad de este método. Por su parte Dabestani et $a^{20}$ validaron los patrones de velocidad de flujo de la AP y encontraron que un TAP $\leq 100$ ms correspondía a PAP elevada (sensibilidad 78\%, especificidad $100 \%$ ). Este método es menos preciso que las estimaciones derivadas de RT, especialmente a frecuencias cardíacas altas o bajas ${ }^{10}$ En el presente trabajo encontramos para el TAP limitaciones relacionadas con FC elevadas. Respecto a las ecuaciones derivadas a partir de la RT, el método de Chemla et al $^{6}$ reportaba los inconvenientes de la imposibilidad o subestimación de la velocidad máxima de la RT y/o medición errada de la $\mathrm{PAD}^{10}$. En este estudio pudimos incluir todos los pacientes, con alta correlación entre los métodos (Chemla-Aduen). Por su parte Aduen et al, reportaron en su trabajo superioridad en su método al encontrar una diferencia media de los valores de PMAP con respecto al CCD de -1.6, menos que la PSAP obtenida tradicionalmente con RT(-3.6) y comparándolo con el método de RP (-13.9) ${ }^{7}$. En un trabajo retrospectivo realizado recientemente donde compararon los 3 métodos analizados en este estudio, entre otros, con mediciones obtenidas invasivamente, encontraron superioridad en la ecuación de Aduen ${ }^{25}$. También cuando este autor comparó su método, la ecuación de Chemla y ecuación de Syyed) con las mediciones obtenidas invasivamente, encontró una discreta superioridad en su método ${ }^{17}$. En este estudio no aplicamos la ecuación derivada de RP (Masuyama et al $)^{1}$ porque su registro fue posible en menos del $60 \%$ de la muestra analizada.

En la segunda etapa de nuestra investigación, encontramos muy alta correlación al comparar los valores de PMAP obtenidos por CCD y la ecuación de Chemla en las 6 etapas del TRVP (mostramos en el estudio t0. t30 y tR). Evidenciamos correspondencia como la señalada por este autor: que valores de PAP>30 mmHg corresponden a PMAP> $20 \mathrm{mmHg}$, representando aproximadamente el $60 \%$ de la PSAP de manera constante ${ }^{3}$. No se dispone en la actualidad de estudios que reporten mediciones comparativas invasiva y no invasiva de la PMAP en el TRVP por considerarse el CCD el estándar de oro para su realización 9,26 . Basándonos en los resultados alentadores de este trabajo, recomendamos desarrollar estudios con mayor población de pacientes que estimen por medio de la ecocardiografía y CCD la PMAP y otras variables involucradas.

\section{Conclusiones:}

Disponemos de ecuaciones derivadas de la RT efectivos y confiables para estimar la PMAP en un amplio grupo de pacientes. El Método de Chemla es útil y preciso en el TRVP.

Agradecimientos: A Dios por darnos la vida y poner- 
nos en el camino donde marchan los sensibles con alto sentido de vocación y servicio. A la Dra. Rita Tamasaukas por la asesoría en los análisis estadísticos, revisión y corrección del manuscrito. Al equipo Médico,
Técnicos en ecocardiografía y enfermería que laboran en los Departamentos de Cardiología y Terapia Intensiva del Hospital Militar Universitario "Dr. Carlos Arvelo".

\section{Referencias}

1. BENAVIDES-LUNA H. Fisiopatología de la hipertensión arterial pulmonar Rev Colomb Cardiol. 2017;24(1):11---15

2. GORTER T, VAN VELDHUISEN J, BAUERSACHS J, BORLAUG B, CELUTKIENE J, COATS S et al. Right heart dysfunction and failure in heart failure with preserved ejection fraction:mechanisms and management. Position statement on behalf of the Heart FailureAssociation of the European Society of Cardiology. Eur J Heart Failure 2018; 20:16-37.

3. VONK NOORDEGRAAF A, WESTERHOF BE, WESTERHOF $\mathrm{N}$. The relationship between the right ventricle and its load in pulmonary hypertension. J Am Coll Cardiol 2017;69:236-243.

4. MASUYAMA T, KODAMA K, KITABATAKE A, SATO $\mathrm{H}$, NANTO S AND INOUE M. Continuous-wave Doppler echocardiographic detection of pulmonary regurgitation and its application to noninvasive estimation of pulmonary artery pressure. Circulation. 1986; 74: 484-492

5. KITABATAKE A, INOUE M, ASAO M, MASUYAMA T, TANOUCHI J, MORITA T. et al. Noninvasive evaluation of pulmonary hypertension by a pulsed Doppler technique. Circulation. 1983; 68: 302-309

6. CHEMLA D, CASTELAIN V, HUMBERT M, HÉBERT J, SIMONNEAU G, LECARPENTIER, et al. New formula for predicting mean pulmonary artery pressure using systolic pulmonary artery pressure. Chest. 2004; 126: 1313- 1318.

7. ADUEN J, CASTELLO R., LOZANO M, HEPLER G, KE-
LLER C.A, ALVAREZ F. et al. An alternative echocardiographic method to estimate mean pulmonary artery pressure: diagnostic and clinical implications. J Am Soc Echocardiogr. $2009 ; 22: 814-9$

8. MITCHELL C, RAHKO P, CANADAY B, FINSTUEN J, FOSTER M, HORTON K et al. Guidelines for Performing a Comprehensive Transthoracic Echocardiographic Examination in Adults: Recommendations from the American Society of Echocardiography JASE 2019; 32(1):1-64

9. RUDSKI L, LAI W, AFILALO J, HUA L, HANDSCHUMACHER M, CHNDRASEKARAN K et al. Guildelines for the Echocardiographic Assessment of Right Heart in Adults: A Report from the American Society of Echocardiography, a registered branch of the European Society or Echocardiography. J Am Soc Echocardiogr. 2010; 23(7):685-713

10. PARASURAMAN $S$, WALKER $S$, LOUDON B GOLLOP N, WILSON A, LOWERY C et al. Michael Assessment of pulmonary artery pressure by echocardiography-Acomprehensive review. IJC Heart \& Vasculature ,2016;12: 45-51

11. YOCK PG, POPP RL. Noninvasive estimation of right ventricular systolic pressure by Doppler ultrasound in patients with tricuspid regurgitation. Circulation. 1984;70: 657-62.

12. MAHAN G, DABESTANI A, GARDIN J, ALLFIE A, BURN C, HENRY W. Estimation of pulmonary artery pressure by pulsed Doppler Echocardiography. Circulation. 1983; 68 (Suppled 3):III- 367(abst). 
13. VELAZCO L. Rol de la ecocardiografía en el estudio de la Hipertensión arterial Pulmonar. Avances Cardiol 2009; 29(2):154-164.

14. REUTER D, HUANG C, EDRICH T. Cardiac output monitoring using indicator-dilution techniques: basics, limits and perspectives.Anesth Analg.2010:799-811.

15. DAVID S. Celermajer Echocardiographic and right heart catheterization techniques in patients with pulmonary arterial hypertension Int J Cardiol. 2008; 125(3):294-303.

16. CARRILLO A, FIOL M, RODRÍGUEZ A. El papel del catéter de Swan-Ganz en la actualidad Med Intens 2010; 34 (3):203214.

17. ADUEN J, CASTELLANO R, DANIELS J, DIAZ J, SAFFORD R, HECKMAN $M$ et al. Accuracy and Precision of Three Echocardiographic Methods for Estimating Mean Pulmonary Artery Pressure. https://doi.org/10.1378/chest.10-0126.

18. RISTOW B, ALI S, REN X, WHOOLEY MA, SCHILLER NB. Elevated pulmonary artery pressure by Doppler echocardiography predicts hospitalization for heart failure and mortality in ambulatory stable coronary artery disease: the Heart and Soul Study. J Am Coll Cardiol. 2007; 49: 43-49

19. HIMELMAN RB, STULBARG M, KIRCHER B, LEE E, KEE L, DEAN NC, et al. Noninvasive evaluation of pulmonary artery pressure during exercise by saline-enhanced Doppler echocardiography in chronic pulmonary disease. Circulation. 1989; 79: 863-871

20. DABESTANI A, MAHAN G, GARDIN JM, TAKENAKA K, BURN C, ALLFIE A, HENRY WL. Evaluation of pulmonary artery pressure and resistance by pulsed Doppler echocardiography. Am J Cardiol. 1987; 59: 662-668.

21. CHAN K, CURRIE P, SEWARD J, HAGLER D, MAIR D, TAJIK A. Comparison of three Doppler ultrasound methods in the prediction of pulmonary artery pressure. J Am Coll Cardiol. 1987; 9: 549-554.

22. SIMONSON J, SCHILLER N. Sonospirometry: a non-invasive method for estimation of mean right atrial pressure based on two dimensional echocardiographic measurements of the inferior vena cava during measured inspiration. J Am Coll Cardiol. 1988; 11: 557-564.

23. FRIEDBERG M, FEINSTEIN J, ROSENTHAL D. A novel echocardiographic Doppler method for estimation of pulmonary arterial pressures. J Am Soc Echocardiogr. 2006; 19: 559-562.

24. ABBAS A, FORTUIN F, SCHILLER N, APPLETON C, MORENO C AND LESTER S. Echocardiographic determination of mean pulmonary artery pressure. Am J Cardiol. 2003; 92: 1373-1376

25. HELLENKAMP K, UNS $€$ OLD U, MUSHEMI-BLAKE S, SHAH A, FRIEDE T, HASENFUß T et al Echocardiographic Estimation of Mean Pulmonary Artery Pressure: A Comparison of Different Approaches to Assign the Likelihood of Pulmonary Hypertension.. J A Soc Echocardiogr.2018;31(1):89-98

26. SANTOS L. CONTRERAS A. MORENO A. MEDINA L. RODRÍGUEZ N. It is useful to evaluate pulmonary vascular reactivity of echocardiography in the pulmonary hypertension?. A challenge to solve. Carta al editor. Arch Cardiol Mex 2017;87: 260-2 